\title{
SELF-ADAPTIVE APSO ALGORITHM FOR SOLVING DIGITAL SIGNATURE RECOGNITION TASK
}

\author{
Alexandru-Daniel STAN \\ Bucharest University of Economic Studies, Romania \\ alexandru.stan1@yahoo.com \\ Cristian Răzvan USCATU \\ Bucharest University of Economic Studies, Romania \\ cristian.uscatu@ie.ase.ro
}

\begin{abstract}
The paper proposes a new method that combines the self-adaptation mechanism and Adaptive Particle Swarm Optimization (APSO) algorithm for binary image registration, taking into consideration the problem of a particular component of banking security which involves the client's signature. The main aim is to register two binary images, considering the affine perturbation model that consists of a mixture of shear and rigid deformation. In most of the cases the image to be recognized is different from the stored image, from geometrical point of view. A self-adaptive APSO method was developed to align the input image to the target one. The light intensity of an individual is defined in terms of mutual information that is calculated between the transformed image and the target image. The proposed registration methodology is evaluated using qualitative and quantitative measures. In the final part of the paper, the experimental results are reported together with some concluding remarks regarding the quality of the resulted methods.
\end{abstract}

Keywords: Self-Adaptive APSO, Image Registration, Mutual Information, Digital Signature. JEL classification: C63

DOI: $10.12948 / \mathrm{ie} 2019.01 .04$

\section{Introduction}

Image registration is an important step in image processing applications and is used in various systems and algorithms including image recognition, computer vision, medical imaging and target recognition [1-4]. The main aim is to align multiple scenes from images acquired at different times, from different viewpoints, different depths, and using different sensors into a single integrated image. Most common types of variations that appear in observed images are: spatial variations, volumetric variations, region of interest variations. In most of the cases the variations in the analyzed images are due acquisition and can be removed using spatial transformation [1-2]. In the following we will work under the assumption that the variations are spatial and are of affine type. In the literature, based from perturbation model, various classes of image registration techniques have been presented such as: landmark-based registration, multi-resolution registration, principal axes transformation (PAT), boundary registration, optimization-based registration, adaptive methods and evolutionary-based approaches [5-9]. The goal of this paper is to develop a new image registration methodology for signature recognition and to improve the Accelerated Particle Swarm Optimization (ASPO) registration method presented in [10]. The proposed methodology belongs to evolutionarybased class and uses the bio-inspired APSO approaches whose parameters automatically adapt and evolve. It addresses the problem of security systems based on digital signatures, but it can be applied to all binary images represented by sparse matrices. The movement between two 
www.conferenceie.ase.ro

individuals takes into consideration the space bounds and the quality of the solutions [10]. The light intensity of the individual is defined in terms of quality measure and uses the mutual information metric.

\section{The proposed methodology}

In geometry, an affine spatial transformation or affinity is a function in affine space that preserves collinearity and ratios of distances [11]. Expansion, scale, geometric contraction, reflection, rotation, shear, translation, similarity transformation, spiral similarities and their combinations are affine transformations. The general affine transformation is a composition of rotations, translations, scale and shears.

In our approach we will take into consideration the rigid transformation with shear effect along $O_{x}$ axe. Taking into consideration the input image $I_{0}$ and the parameters $(a, b, s, \theta, d)$ the output image is described as follows:

$$
\left(\begin{array}{l}
x^{1} \\
y^{1}
\end{array}\right)=\left(\left[\begin{array}{ll}
1 & d \\
0 & 1
\end{array}\right] \cdot\left(\left[\begin{array}{l}
a \\
b
\end{array}\right]+s \cdot\left[\begin{array}{cc}
\cos \theta & -\sin \theta \\
\sin \theta & \cos \theta
\end{array}\right] \cdot\left[\begin{array}{l}
x \\
y
\end{array}\right]\right)\right)
$$

The APSO developed by Xin-She Yang [12] represents a special case of firefly algorithm and belongs to meta-heuristic nature inspired class of algorithms. Typically it is used to solve a range of optimization problems in real-world applications, from shaded PV Systems to Network Plan Optimization, from image processing to global numerical optimization $[13,14]$. In the standard firefly algorithm, the attractiveness, the movement and the distance represents the main features. The movement of a firefly $I$ toward a brighter firefly $j$, is defined by:

$$
x_{i}^{\prime}=x_{i}+\beta_{0} e^{-\gamma r_{i j}^{2}}\left(x_{j}-x_{i}\right)+\alpha \cdot \varepsilon
$$

where $x_{i}$ represents the current position of firefly $i$, the $\beta_{0}$ represents the attractiveness constant when distance between two fireflies $r_{i j}$ is 0 , the third term represents the nonlinear attractiveness which varies with distance, while the last term represents the random walk with $\alpha$ the randomization parameter, and $\varepsilon$ a random number.

If we replace $x_{j}$ with the current global best solution $g$ then the above equation becomes a variant of accelerated particle swarm optimization (APSO) [15].

$$
x_{i}^{\prime}=x_{i}+\beta_{0} e^{-\gamma r_{i j}^{2}}\left(g-x_{i}\right)+\alpha \cdot \varepsilon
$$

The idea that stands behind using global best solution $g$ is primarily to increase the diversity in the quality solutions. In our methodology, we use two updating rules [10]:

$$
\begin{gathered}
x_{i}^{\prime}(k)=x_{i}(k)+\beta_{0} e^{-\gamma r(k)_{i j}^{2}}\left(g(k)-x_{i}(k)\right)+\frac{h b(k)-l b(k)}{\max _{k}(h b(k)-l b(k))} \cdot \varepsilon \cdot c \\
x_{i}^{\prime}(k)=x_{i}(k)+\beta_{0} e^{-\gamma r(k)_{i j}^{2}}\left(g(k)-x_{i}(k)\right)+\frac{h b(k)-l b(k)}{\max _{k}(h b(k)-l b(k))} \cdot \varepsilon \cdot c \cdot \exp (1-\text { fitness }(g))
\end{gathered}
$$

where $l b(k), h b(k)$ represents the bounds of the parameter $k, \mathrm{c}$ is a given constant scale factor, $\varepsilon$ represents a random number generated from uniform distribution $U(0,1)$ and fitness $(g)$ represent the quality of the attractor. The distance between two fireflies is defined by 
www.conferenceie.ase.ro

$$
\begin{gathered}
r_{i j}(k)=\frac{\left|x_{i}(k)-x_{j}(k)\right|}{\operatorname{Dmax}(k)} \\
\operatorname{Dmax}(k)=\frac{h b(k)-l b(k)}{\sqrt{D}}
\end{gathered}
$$

where $D$ represents the number of components of fireflies.

Comparing with many other bio-inspired algorithms, the APSO uses tree parameters, $\beta_{0}, \gamma, \alpha$, with a simple mechanism. The initial values of parameters represents the most challenging issue in meta-heuristic algorithms, as they control the convergence and the accuracy of the algorithm. By setting appropriate parameter values, the performance of the algorithm is positively influenced.

The input parameters $\beta_{0}, \gamma, \alpha$ of standard algorithm influence the results. For example, in APSO, the parameter $\gamma$ represents the influence range. A small value of $\gamma$ is similar with high visibility between fireflies while a higher value of $\gamma$ is similar with low visibility. If $\gamma=0$ all the fireflies belong to a single swarm. A higher value $\gamma$ can lead to a random walk, the idea of swarm being dissolved. The diversity and properties of the entire population is influenced by $\gamma$. Also, the parameter $\beta_{0}$ impacts the convergence of the algorithm. If the value of $\beta_{0}$ is 0 the entire process becomes a random walk. In essence, the parameter tuning represents an optimization problem which requires higher-level optimization methods.

Parameter values set at the beginning of optimization process can become inappropriate in later generations, leading to bad results. A solution for this is to allow the parameters $\beta_{0}, \gamma, \alpha$ to vary according to each firefly experience and current situation. Those parameters are encoded into genotype of individuals as follows:

$$
x=\left(x_{1}, \ldots, x_{n}, \beta, \sigma_{1}, \gamma, \sigma_{2}, \alpha, \sigma_{3}\right)
$$

where $x_{1}, \ldots, x_{n}$ represent the solution part, parameters $\beta, \gamma, \alpha$ represent the current values of control parameters, and $\sigma_{\beta}, \sigma_{\gamma}, \sigma_{\alpha}$ represent the standard deviations of the control parameters. The values of control parameters are changing according to standard uncorrelated mutation with $n$ step [16]. The mutation is defined by the following equations:

$$
\begin{gathered}
\sigma_{i}^{\prime}=\sigma_{i} \cdot e^{\left(\tau^{\prime} \cdot N(0,1)+\tau \cdot N_{i}(0,1)\right)}, i=1, \ldots 3 \\
\beta^{\prime}=\beta+\sigma_{1}^{\prime} \cdot N(0,1) \\
\gamma^{\prime}=\gamma+\sigma_{2}^{\prime} \cdot N(0,1) \\
\alpha^{\prime}=\alpha+\sigma_{3}^{\prime} \cdot N(0,1)
\end{gathered}
$$

where $\tau^{\prime}$ and $\tau$ denote the learning rate with $\tau^{\prime} \propto 1 / \sqrt{2 n}$ and $\tau \propto 1 / \sqrt{2 \cdot \sqrt{n}}$. To prevent the use of very small mutation strengths, a threshold rule is applied:

$$
\sigma_{i}^{\prime}<t h \text { then } \sigma_{i}^{\prime} \leftarrow t h
$$


Starting from the particular affine model (1), the image registration task is to compute a function $f(x, y)$ such that $I_{1}\left((f(x, y))^{T}\right)=I_{0}\left(x^{1}, y^{1}\right)$. The function $\mathrm{f}$ is completely defined by the parameters $(a, b, s, \theta, d)$ :

$$
f_{a, b, s, \theta, d}(x, y)=\frac{1}{s} \cdot R^{T} \cdot\left[\left(\begin{array}{cc}
1 & -d \\
0 & 1
\end{array}\right) \cdot\left(\begin{array}{l}
x \\
y
\end{array}\right)-\left(\begin{array}{l}
a \\
b
\end{array}\right)\right]
$$

where $R$ represents the rotation matrix with $R^{-1}=R^{T}$.

In our approach, the parameters that self-adapt are $\beta_{0}, \gamma$. The firefly $x$ becomes:

$$
x=\left(a, b, s, \theta, d, \beta, \sigma_{\beta}, \gamma, \sigma_{\gamma}\right)
$$

Let $n$ be the number of fireflies; MaxGeneration the maximum number of generations; $\beta_{0}, \gamma$ the initial attractiveness and light absorption coefficient; $\sigma_{\beta}, \sigma_{\gamma}$ the initial dispersion; $t h$ the threshold value, $h b$ and $l b$ the bounds for firefly components; $r$ a parameter that controls the desired sub-optimal fitness value, $r<1$. The proposed firefly algorithm is presented as follows:

Step 1. Randomly generate the initial population $X_{0}=\left\{x_{1}, x_{2}, x_{3}, \ldots, x_{n}\right\}$

Step 2. Compute $L_{i}$ the light intensity for each $x_{i} \in X_{0} ; t \leftarrow 0$;

Step 3. while $(t<$ MaxGeneration \&\& fitness $(g)<\mathrm{r})$

Step 3.1 Compute $g$, the best fitted firefly

Step 3.2 for $i=1: n$

Step 3.2.1 Compute the $\sigma_{i \beta}, \sigma_{i \gamma}, \beta_{i}, \gamma_{i}$

Step 3.2.2 Move firefly $x_{i}$ to firefly $g$

Step 3.3 Evaluate all candidate solutions and update light intensity

Step $3.4 t \leftarrow t+1$

Step 4. Rank all fireflies and return the best fitted firefly

\section{Experimental Results}

In order to measure the accuracy and effectiveness of the proposed methodology we have performed an extensive testing work on various binary digital signature images. The quality of the proposed algorithm is measured using Success Rate (SR), Mutual Information Rate (MIR), Signal-to-Noise Ratio (SNR), Root Mean Squared Error (RMSE), and Root Mean Squared Signal-to-Noise Ratio (RMS-SNR) [10].

The reported results correspond to a set of 16 pairs (sensed image, target image), denoted as $P J$, representing signatures. The results are obtained by running the algorithm 500 times for each input pair of images. Note that the first APSO algorithm, APSO1, uses the updating rule (4) and the following input parameters: the population size $n=50$, MaxGeneration $=$ $200, r=0.85, \beta_{0}=2, \gamma=2, \sigma_{\beta}=1, \sigma_{\gamma}=1$.The second APSO algorithm, APSO2, uses the updating rule (5) and the same input parameters.

Table 1. Experimental results

\begin{tabular}{|c|c|c|c|c|c|c|c|c|c|c|}
\hline & \multicolumn{4}{|c|}{ APSO1 } & \multicolumn{5}{c|}{ APSO2 } \\
\hline Input & SR & MI & RMSE & RMS & SNR & SR & MI & RMSE & RMS & SNR \\
\hline$P J_{1}$ & $98.4 \%$ & 0.863 & 0.064 & 15.758 & 23.839 & $99.80 \%$ & 0.872 & 0.062 & 16.059 & 24.057 \\
\hline$P J_{2}$ & $99.4 \%$ & 0.866 & 0.050 & 20.148 & 26.031 & $100 \%$ & 0.871 & 0.049 & 20.416 & 26.161 \\
\hline
\end{tabular}


www.conferenceie.ase.ro

\begin{tabular}{|l|c|c|c|c|c|c|c|c|c|c|}
\hline$P J_{3}$ & $95.2 \%$ & 0.849 & 0.063 & 16.562 & 24.208 & $98.60 \%$ & 0.865 & 0.059 & 16.804 & 24.466 \\
\hline$P J_{4}$ & $97.4 \%$ & 0.854 & 0.062 & 16.478 & 24.200 & $99.20 \%$ & 0.869 & 0.059 & 16.878 & 24.498 \\
\hline$P J_{5}$ & $99.6 \%$ & 0.87 & 0.061 & 16.472 & 24.274 & $100 \%$ & 0.873 & 0.060 & 16.481 & 24.299 \\
\hline$P J_{6}$ & $80.2 \%$ & 0.737 & 0.082 & 16.174 & 23.195 & $89.60 \%$ & 0.803 & 0.068 & 17.462 & 24.297 \\
\hline$P J_{7}$ & $93 \%$ & 0.819 & 0.069 & 16.401 & 23.907 & $99.60 \%$ & 0.869 & 0.057 & 17.393 & 24.767 \\
\hline$P J_{8}$ & $87.8 \%$ & 0.801 & 0.061 & 17.092 & 24.537 & $88.60 \%$ & 0.839 & 0.058 & 17.235 & 24.615 \\
\hline$P J_{9}$ & $99.4 \%$ & 0.866 & 0.062 & 16.075 & 24.062 & $100 \%$ & 0.873 & 0.060 & 16.382 & 24.246 \\
\hline$P J_{10}$ & $93.6 \%$ & 0.822 & 0.070 & 15.946 & 23.685 & $99.20 \%$ & 0.865 & 0.060 & 16.715 & 24.398 \\
\hline$P J_{11}$ & $90.2 \%$ & 0.846 & 0.057 & 18.197 & 25.055 & $91.40 \%$ & 0.855 & 0.055 & 18.321 & 25.179 \\
\hline$P J_{12}$ & $92.4 \%$ & 0.814 & 0.105 & 10.772 & 20.188 & $98.60 \%$ & 0.861 & 0.088 & 11.288 & 20.945 \\
\hline$P J_{13}$ & $95.6 \%$ & 0.846 & 0.059 & 17.751 & 24.812 & $98.80 \%$ & 0.867 & 0.055 & 18.257 & 25.179 \\
\hline$P J_{14}$ & $76.6 \%$ & 0.690 & 0.090 & 15.923 & 22.788 & $89.40 \%$ & 0.790 & 0.068 & 17.979 & 24.471 \\
\hline$P J_{15}$ & $83 \%$ & 0.749 & 0.086 & 15.318 & 22.804 & $96.40 \%$ & 0.843 & 0.063 & 17.051 & 24.405 \\
\hline$P J_{16}$ & $83.4 \%$ & 0.739 & 0.072 & 18.672 & 24.472 & $93.80 \%$ & 0.824 & 0.056 & 20.660 & 25.892 \\
\hline
\end{tabular}

From the accuracy point of view, the results of APSO2 algorithm are better or in some cases, significantly better than the APSO1 variant. Taking into consideration that the input images are the same, the proposed self-adaptive APSO outperforms in some cases in both variant the APSO without self-adapting. For example, in [10] using APSO1 and APSO2 the lower value of SR is obtained for picture \#14 with $S R=71 \%$ and $S R=73.75 \%$.Using the self-adaptive variant of APSO, the results improve from both accuracy and efficiency point of view.

\section{Conclusions}

In this paper we proposed a methodology based on self-adaptive APSO algorithm for image registration. Starting from a sensed image, the idea is to align it with a target image using the mutual information measure that computes the similarities between images. This method can be applied on any gray-scale images. The effectiveness of our proposed methodology has been established by implementing the self-adaptive mechanism for input parameters.

We have conducted a series of tests to evaluate the quality and accuracy of obtained results. The tests point out that the algorithm is very fast and in most of the cases, the accuracy is fairly good, better than non-adaptive algorithm.

\section{References}

[1] J. Modersitzki, Numerical Methods for Image Registration, New York: Oxford University Press, 2004.

[2] A.A. Goshtasby, Image Registration: Principles, Tools and Method. London: Springer Science \& Business Media, 2012.

[3] G. Hermosillo et al., "Image Registration in Medical Imaging: Applications, Methods, and Clinical Evaluation", Multi-Modality State-of-the-Art Medical Image Segmentation and Registration Methodologies, vol. 2, pp.263-313, February 2011.

[4] S. Uchida, "Image processing and recognition for biological images", Development, Growth \& Differentiation, vol. 55, no. 4, pp. 523-549, 2013.

[5] C. Davatzikos, J. L. Prince, R. N. Bryan, "Image registration based on boundary mapping", IEEE Transactions on Medical Imaging, vol.15, no. 1, pp. 112-115, 1996. 
www.conferenceie.ase.ro

[6] S. Nandish, "Multiresolution image registration for multimodal brain images and fusion for better neurosurgical planning", Biomedical Journal, vol. 40, no. 6, pp. 329-338, 2017.

[7] N.M. Alpert, J.F. Bradshaw, D. Kenedy, J. Correia, "The Principal Axes TransformationA Method for Image Registration", Journal of Nuclear Medicine, vol. 31, no.10, pp. 1717$1722,1990$.

[8] S. Klein, M. Staring, J. P. W. Pluim, "Evaluation of Optimization Methods for Nonrigid Medical Image Registration Using Mutual Information and B-Splines", IEEE Transactions on Image Processing, vol. 16, no. 12, pp.2879-2890, 2007.

[9] C. Cocianu, A. Stan, "New Attempts in Binary Image Registration", in 2018 5th International Conference on Control, Decision and Information Technologies (CoDIT), vol. 1, Thessaloniki, Greece, 2018, pp. 253-258.

[10] C. Cocianu, A. Stan, "New Evolutionary-Based Techniques for Image Registration", Applied Sciences, vol. 9, no. 1, pp.176-191.

[11] L. Brown, "A Survey of Image Registration Techniques", ACM Comput. Surv., vol. 24, no. 4, pp. 325-376, 1992.

[12] X.S. Yang, Nature-inspired metaheuristic algorithms, Frome, Luniver, 2008.

[13] S. Rajendran , H. Srinivasan , "Simplified accelerated particle swarm optimization algorithm for efficient maximum power point tracking in partially shaded photovoltaic systems", IET Renewable Power Generation, vol. 10, no. 9, pp.1340-1347, 2016.

[14] H. Zhaolan, Z. Yang, "Accelerated Particle Swarm Optimization to Solve Large-Scale Network Plan Optimization of Resource-Leveling with a Fixed Duration", Mathematical Problems in Engineering, vol. 2018, pp. 1-11, 2018.

[15] X.S. Yang, Cuckoo Search and Firefly Algorithm, Springer: London, UK, 2014.

[16] A. Eiben, J. Smith, Introduction to Evolutionary Computing, Berlin: Springer Berlin Heidelberg, 2003. 